\title{
Packing dynamics of spherical and nonconvex grains sedimenting at low Stokes number
}

\author{
Antonio Olmedilla, ${ }^{*}$ Miha Založnik, ${ }^{\dagger}$ Thomas Messmer, ${ }^{\ddagger}$ Bernard Rouat, ${ }^{\S}$ and Hervé Combeaul \\ Institut Jean Lamour, CNRS-Université de Lorraine, Nancy, France
}

(Received 19 September 2018; published 29 January 2019)

\begin{abstract}
This paper addresses the phenomenon of random packing of sedimenting grains in the context of metallic alloy solidification from a dynamics perspective. More precisely, we investigate the evolution of the grains from the initial steady-state sedimentation until the final mechanical equilibrium—packing—is achieved. The packing dynamics of two grain geometries (spherical and nonconvex) is investigated for a hydrodynamic condition of a low ratio of grain inertia to viscous dissipation, given by a low Stokes number, St, of $o\left(10^{-3}\right)$, which is typical in solidification. An experimental setup that consists of a vertical column filled with glycerol is employed to reproduce the packing of the sedimenting grains by means of two different protocols (sequential and collective), enabling to track the trajectory of the grains. The grain packing is also simulated by means of a discrete element method for both grain geometries in case of collective packing protocol, complementing the experimental work - trajectory tracking - with the time evolution of the local solid fraction, of the local number of contacting neighbors and of the grain orientation during the packing dynamics.
\end{abstract}

DOI: 10.1103/PhysRevE.99.012907

\section{INTRODUCTION}

In solidification of metal alloys, the solid grains contained in the melt sediment due to the slight density difference between the solid and liquid. The characteristic geometries of the sedimenting grains are globular (spherelike) and dendritic (nonconvex). This grain sedimentation forms a complex packing network. This phenomenon is present in a wide range of solidification processes such as the aluminium direct chill casting [1] or the steel static ingot casting [2]. The understanding of the formation of this packing structure, i.e., how the grains decelerate and rearrange from a sedimentation regime to packing is fundamental for a better comprehension of the mushy zone in solidification, which has an important impact on the solidification structure and defects of the material, which are related with the final mechanical properties of the casting product [3].

The phenomenon of packing of sedimenting grains in a viscous fluid can be observed from two different perspectives: (i) statics, once the particles have achieved the mechanical equilibrium - grain packing — and (ii) dynamics, which is the transition from the steady-state sedimentation to the final mechanical equilibrium of the grains. In the first perspective, the main question for the grain packing in the solidification context is that of the packing fraction. Plenty of information is available in literature about the particle packing question, mainly for spheres [4-9] and nonspherical convex grain packing [10]. Scarse information is available about the packing of nonconvex grains (such as the nonconvex dendritic grains

\footnotetext{
*antonio.olmedilla.aero@gmail.com

†miha.zaloznik@univ-lorraine.fr

¥thomas.messmer5@etu.univ-lorraine.fr

§bernard.rouat@univ-lorraine.fr

"herve.combeau@univ-lorraine.fr
}

in solidification) and it is exclusively focused on dry packings [11-13]. As far as we know, the packing of nonconvex grains by sedimentation in the presence of a viscous fluid has been exclusively addressed in Ref. [14], where the packing fraction for nonconvex dendritic grains ranges from approximately 0.39 up to 0.63 depending on the grain geometry and the hydrodynamic conditions of the packing.

For the dynamics perspective, the main question is that of understanding the motion that the grains undergo before achieving the mechanical equilibrium-packing. First we focus on the case of sequential packing of sedimenting grains in presence of a viscous liquid. The grain trajectory during the motion toward to the previously packed particles is influenced by the viscous fluid that dissipates part of the initial kinetic energy of the grain. This dissipative effect has been experimentally shown by investigating the approach of a sphere to a wall [15]. Similar results are obtained for two approaching spheres [16]. The amount of dissipated kinetic energy is governed by the ratio of grain inertia to viscous dissipation, that is given by the Stokes number, St. In the case of a Stokes number lower than approximately 10, the initial kinetic energy of the particle is fully dissipated by the viscous fluid, avoiding the particle rebound [17]. Under low Stokes conditions, the repulsive force that a particle undergoes when it approaches to a smooth wall or to another particle when immersed in a viscous fluid corresponds to the lubrication drag force. A preliminary model of the lubrication drag force is given by the creeping-flow equations of the fluid for the near-wall region. Corrective terms to the repulsive force obtained by the creeping-flow equations can be considered to extend the validity of the model to a much wider region [18], to account for the inertial effects [19], for the effect of surface roughness [20], etc.

The situation of collective packing of sedimenting grains in the presence of a viscous liquid under low Stokes is much more complex than that of sequential packing, where only 
one particle is moving towards others that are already in mechanical equilibrium. In the collective case, lots of grains are in motion simultaneously. In this way, not only does the dissipative effect of the fluid at the lubrication region for every binary particle collision play a role (as for the sequential case) but also the ability the granular system has to jam. In this way, the interparticle collisions play a fundamental role. The dynamics of collective granular packing is addressed in the context of cake formation for spherical particles $[21,22]$. However, as far as we know, no information is available in case of nonspherical nonconvex grains.

In this paper, we first present a simple model of the packing dynamics of sedimenting spherical grains in case of sequential packing that enables us to show the dimensionless numbers that govern this phenomenon and to plot an approximate trajectory of the grains. Subsequently, we use an experimental setup based on a hydrodynamic similarity with the actual phenomenon of packing of sedimenting grains in the context of solidification [St of $o\left(10^{-3}\right)$ ] to investigate the motion of grains (both spherical and nonconvex dendritic geometries) during the transition. Experiments for both sequential and collective packing protocols are carried out, showing the important effect that collectivity plays on the packing dynamics. To complement our experimental results, we numerically simulate the packing dynamics of both spheres and nonconvex dendritic grains in case of collective packing by means of an in-house discrete element method (DEM) model. With this numerical tool we obtain the evolution of the local solid fraction, of the number of contacting neighbors and of the grain rotation in Lagrangian specification during the transition from the initial steady-state sedimentation regime to the final packing. In this paper, the grains are growthless, which mimics those situations of grain packing in the solidification context where the size evolution of the grains is negligible when compared to their motion.

\section{MODEL OF PACKING DYNAMICS OF SEQUENTIALLY SEDIMENTING SPHERES}

In this section we identity the dimensionless parameters that govern the packing dynamics of sedimenting spheres for the sequential packing protocol. In the sequential protocol each of the grains of the collection is packed one by one, whereas in the collective protocol a great amount of grains simultaneously packs. Initially each of the grains (of density $\rho_{\text {sph }}$ and diameter $d_{\text {sph }}$ ) moves in the quiescent viscous liquid (of density $\rho_{l}$ and kinematic viscosity $v_{l}$ ) towards those grains that have previously packed (packing front) with a limit velocity of $v_{\mathrm{sph}}^{\mathrm{sed}}=\left(\rho_{\mathrm{sph}} / \rho_{l}-1\right) g_{o} d_{\mathrm{sph}}^{2} /\left(18 v_{l}\right)$, where $g_{o}$ is the gravity. At a certain distance from the packing front the grain decelerates. After it, the grain settles in the first mechanical stable position that it gets [9].

The dynamics of the grain towards its final packing position is governed by the grain weight, the buoyancy, the drag and the lubrication forces. Herein we focus on the grain motion along the gravity direction, z. To get a simple expression for the grain motion, we simplify this phenomenon by adopting the configuration of a smooth sphere moving towards a smooth plane. The vertical coordinate of this plane

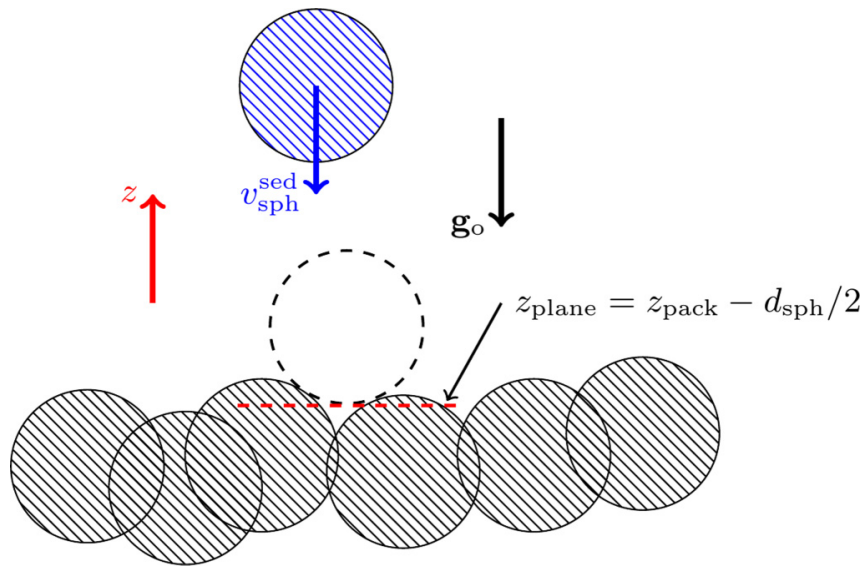

FIG. 1. Schematic representation of the sequential packing of spheres. A spherical grain moves towards the grains that have previously packed (packing front) with an initial steady-state velocity of $v_{\mathrm{sph}}^{\mathrm{sed}}$. After a certain time this grain achieves its final packing position (dashed grain). This phenomenon is simplified by the approach of a sphere to a smooth plane that is schematically drawn (dashed horizontal line). The coordinate $z$ is antiparallel to the gravity direction.

$z_{\text {plane }}$ is related to the vertical coordinate of the grain center at packing $z_{\text {pack }}$ by $z_{\text {plane }}=z_{\text {pack }}-d_{\text {sph }} / 2$ (see Fig. 1 ).

With this simplification, the dimensionless form of the equation of grain motion for the vertical direction reads

$$
\frac{d^{2} z^{*}}{d t^{* 2}}+\frac{2}{\mathrm{St}}\left(1+\frac{1}{2 \xi^{*}}\right) \frac{d z^{*}}{d t^{*}}=-\frac{2}{\mathrm{St}},
$$

where $z^{*}$ is the dimensionless vertical coordinate of the grain center with respect to its packing position $\left(z^{*}=(z-\right.$ $\left.\left.z_{\text {pack }}\right) / d_{s p h}\right), \xi^{*}$ is the dimensionless gap between the sphere and smooth plane that is equal to $z^{*}$, and the dimensionless time by $t^{*}=t /\left(d_{s p h} / v_{s p h}^{\text {sed }}\right)$. The Stokes number $\mathrm{St}$ is given by

$$
\mathrm{St}=\left(\frac{\rho_{\mathrm{sph}}}{\rho_{l}}\right)\left(\frac{d_{\mathrm{sph}} v_{\mathrm{sph}}^{\mathrm{sed}}}{9 v_{l}}\right) .
$$

Note that because of the small density difference between the solid grain and the liquid, $\rho_{\mathrm{sph}} / \rho_{l} \approx 1$, (common in grains solidifying from a liquid metal) we can write the Reynolds number as a function of the Stokes number, $\mathrm{Re} \approx 9 \mathrm{St}$. In this way, the packing dynamics of a sedimenting sphere in case of sequential packing protocol is governed by a single dimensionless number, $\mathrm{St}$, which is the ratio between the grain inertia and the viscous dissipation forces.

Equation (1) is used in next section to provide a reference trajectory (for spheres sequentially packed) with which the experimental work is compared.

\section{EXPERIMENTS}

\section{A. Setup}

An experimental setup is used to investigate the packing dynamics of sedimenting grains for both sequential and collective packing protocols. It consists of a vertical sedimentation cylindrical column with an inner diameter, $D_{\text {in }}$, of $120 \mathrm{~mm}$ filled up with pure glycerol (of density $1262 \mathrm{~kg} / \mathrm{mm}^{3}$ and dynamic viscosity of $1180 \mathrm{mPa}$ s [23]). 

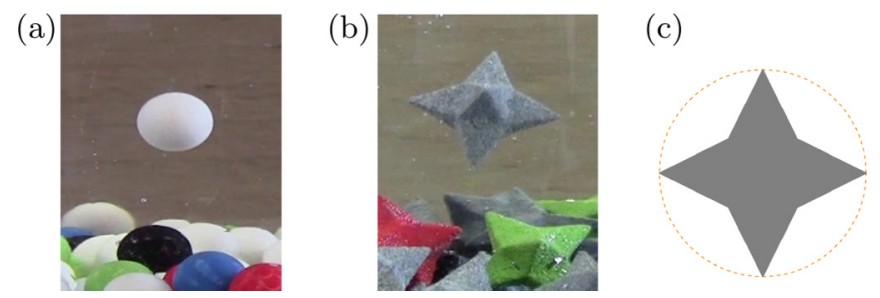

FIG. 2. Snapshot of the packing of sedimenting grains by means of the sequential packing protocol for (a) the spherical grains and (b) the nonconvex dendritic grains; (c) schematic representation of the circumscribing sphere for nonconvex dendritic grains.

Two grain geometries are employed: a spherical to represent the globular grains in solidification and a nonconvex geometry of six principal arms to represent the solidification dendritic grains. The spheres are made of cornstarch with a density of $\rho_{\mathrm{s}}=1391 \pm 6 \mathrm{~kg} / \mathrm{mm}^{3}$ and diameter of $5.92 \pm 0.05 \mathrm{~mm}$. The nonconvex dendritic grains which are based on six quadrilateral pyramids with an apex-angle of 45 degrees are made by additive manufacturing with a bulk density of $\rho_{\mathrm{s}}=1484 \pm 17 \mathrm{~kg} / \mathrm{mm}^{3}$, and equivalent diameter of $d_{\mathrm{eq}}=7.70 \pm 0.31 \mathrm{~mm}$, and a widest grain length (tip-to-tip length passing along the grain center) of $l_{c}=$ $12.59 \pm 0.22 \mathrm{~mm}$. In the case of the spheres, the widest grain length is simply the grain diameter. More details of the setup and grain characteristics are given in [14]. In this way, the grain-to-fluid density ratio remains close to unity with a Stokes number of $\mathrm{St}=o\left(10^{-3}\right)$ for both grain collections, which are conditions typically achieved in metal casting processes.

In the sequential packing protocol, the grains are poured manually one by one in the liquid, where they sediment and pack over an existing bed of packed grains [see Figs. 2(a) and 2(b) for sequential packing of sedimenting spherical and nonconvex dendritic grains, respectively].

In the collective packing protocol, the grains are poured to the sedimentation chamber of the column from the grain reservoir by means of a hopper (see Fig. 3). An equivalent collective condition between the spherical and the nonconvex dendritic grain collections is desired to compare the packing dynamics of them. Assuming the sedimenting grains are uniformly distributed, we define the circumscribed volumetric fraction of sedimentation, $\phi_{\text {circ }}^{\text {sed }}$, by the volume of the grain circumscribing spheres to the volume of the sedimentation zone. In the case of spherical grains $\phi_{\text {circ }}^{\text {sed }}$ is equivalent to the volumetric solid fraction of sedimentation, $\phi_{\mathrm{s}}^{\text {sed }}$. In the case of the nonconvex dendritic grains the relation between the circumscribed volumetric fraction of sedimentation and the volumetric solid fraction of sedimentation is given by $\phi_{\text {circ }}^{\text {sed }}=\left(l_{c} / d_{\mathrm{eq}}\right)^{3} \phi_{\mathrm{s}}^{\text {sed }}$ [see the circumscribing sphere for the nonconvex dendritic grains in Fig. 2(c)]. We also define the mean free distance between the circumscribing spheres of every couple of neighboring grains in sedimentation as $L_{\text {free }}^{\text {sed }} \approx\left[\left(\phi_{\text {circ }}^{\text {sed }}\right)^{-1 / 3}-1\right] l_{c}$. The hopper permits to control the rate of grain pouring, $\dot{N}$ (and consequently controlling the circumscribing fraction) in the sedimentation chamber of the column. In the experiments the hopper opening was

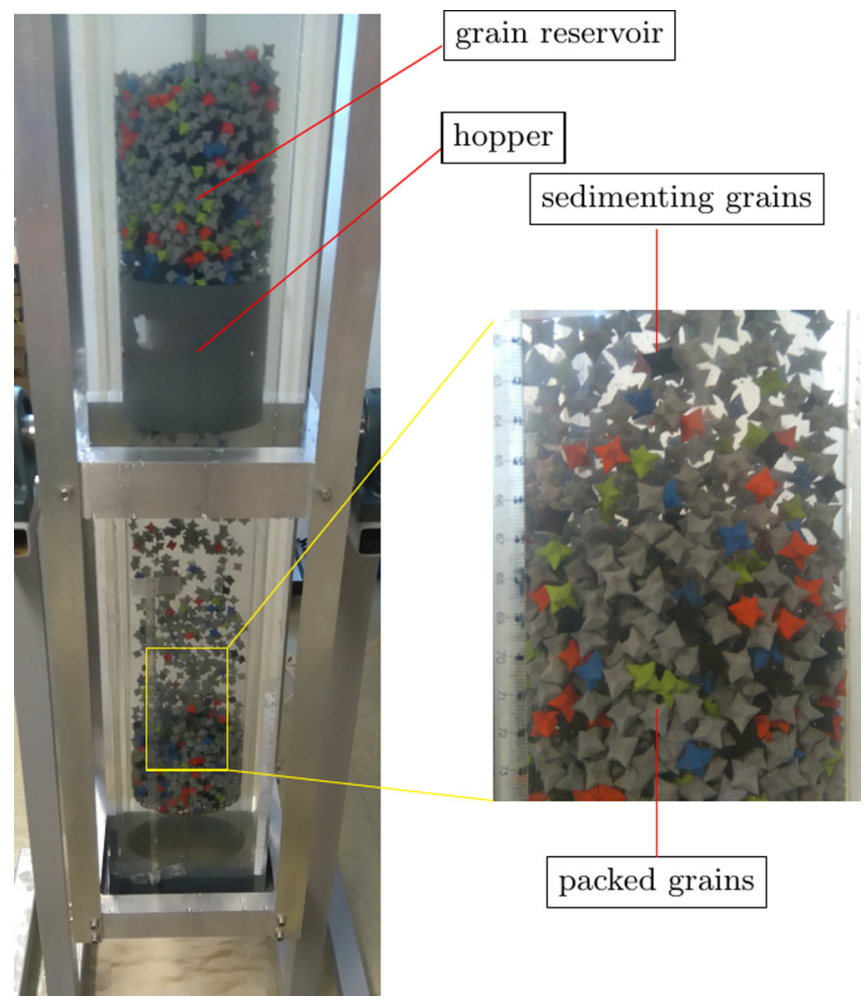

FIG. 3. Snapshot of the packing of sedimenting nonconvex dendritic grains by means of the collective packing protocol. We zoomed the zone where the grains decelerate and pack.

set such that a $\phi_{\text {circ }}^{\text {sed }}$ of approximately 0.20 was achieved for both spherical and nonconvex dendritic grain collections (this corresponds to $\phi_{\mathrm{s}}^{\text {sed }} \approx 0.20$ for the spherical collection and $\phi_{\mathrm{s}}^{\text {sed }} \approx 0.05$ for the dendritic collection). With this condition, $L_{\text {free }}^{\text {sed }}$ is approximately $0.71 l_{c}$ for both spheres and nonconvex dendritic grains.

\section{B. Grain trajectory}

The characterization of the grain motion in the transition from the initial steady-state sedimentation to the final packing is carried out by means of grain visual tracking for both sequential and collective protocols (see Fig. 4 as an example of the collective case for spheres). A fixed HD video camera is used to record the trajectory of the grains projected on the $x-z$ plane. In the case of sequential packing, 10 grains are tracked for each grain geometry. In the case of collective packing, the sample is also composed of 10 grains for each grain geometry. Over $30 \%$ of the system particles are painted to ease the tracking for the collective case.

The projected trajectory of the center of the tracked grains is obtained in a global reference frame fixed to the vessel $\left\{x\left(t_{n}\right), z\left(t_{n}\right)\right\}$ with an acquisition frequency of $1 \mathrm{~Hz}\left(t_{n}=n \Delta t_{a}\right.$ with $n \in \mathbb{Z}^{*}$ and $\Delta t_{a}=1 \mathrm{~s}$ ). The acquirement of the coordinate in the observation direction, $y$, is not possible. A grain is considered in mechanically stable equilibrium (packed) when its position has not perceptable modifications during $5 \mathrm{~s}$. Afterwards, we translate the tracked particle trajectory from the global to its own reference frame $\left[\tilde{z}(t)=z(t)-z_{\text {pack }}\right.$; see the global and grain reference frames in Fig. 4]. We perform 


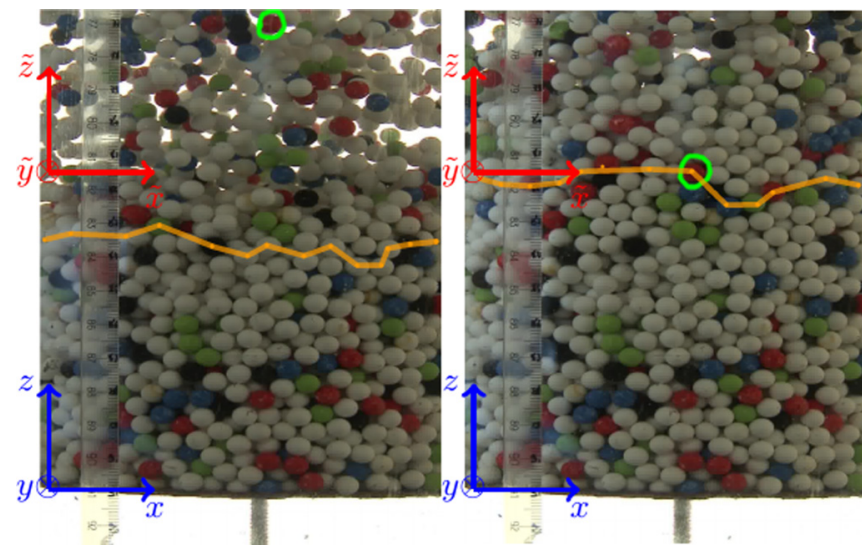

FIG. 4. Grain tracking example for the case of collective packing of sedimenting spheres. The rounded grain is tracked from an initial instant (left) until the final instant of packing, $114 \mathrm{~s}$ after (right). The packing front at a certain instant, that we defined as the virtual surface formed by joining the those grains that just packed, is also shown. The fixed-to-vessel global reference frame $\{x, y, z\}$ is shown and also the fixed-to-vessel particle reference frame $\{\tilde{x}, \tilde{y}, \tilde{z}\}$ whose origin vertical position is located at the packing vertical position of the grain center.

detailed analyses for the final segment of the trajectory, corresponding to $0 \leqslant \tilde{z} \leqslant 6 \bar{d}_{\text {eq }}$. The time origin for each particle is such that $\tilde{z}(\tilde{t}=0)=6 \vec{d}_{\mathrm{eq}}$.

Subsequently, all results are given in dimensionless physical variables, to be able to compare the outcomes of both geometries. The dimensionless center position is $\tilde{\mathbf{x}} / \bar{d}_{\mathrm{eq}}$, and the dimensionless time is $\tilde{t} /\left(\bar{d}_{\mathrm{eq}} / \bar{v}_{z_{o}}\right)$, where $\bar{v}_{z_{o}}$ is the mean vertical velocity at $\tilde{z}=6 \bar{d}_{\mathrm{eq}}$. For each tracked grain, $v_{z_{o}}$ is obtained by the derivation of the vertical coordinate at $\tilde{t}=0$ by means of the central difference scheme:

$$
v_{z_{o}}=\frac{\tilde{z}\left(\tilde{t}=\Delta t_{a}\right)-\tilde{z}\left(\tilde{t}=-\Delta t_{a}\right)}{2 \Delta t_{a}} .
$$

For the sequential packing protocol, the mean evolution of the vertical coordinate over time is shown in Fig. 5 for the spherical and nonconvex dendritic grains. We establish the onset of the transition from the steady-state sedimentation to packing when the repulsive lubrication force due to the presence of the packed grain bed reduces the velocity down to $95 \%$ of its initial value. By means of Eq. (1), it is found to be at a vertical distance of approximately $4.5 d_{\mathrm{eq}}$ with respect to the final packing position.

The first contact with the packed bed is achieved at an instant of $\tilde{t} /\left(\bar{d}_{\mathrm{eq}} / \bar{v}_{z_{o}}\right)$ approximately 6 and 8 for the spheres and nonconvex grains, respectively (see points $\mathrm{A}$ and $\mathrm{B}$ in Fig. 5). After it, the grain rearranges until achieving the final packing position.

In this way, we subdivide the transition from the initial steady-state to the final mechanical equilibrium (packing) into two stages: stage I, which is characterized by the deceleration of the sedimenting grain without interparticle collisions (prior to the first contact), and stage II, which is characterized by the contact interactions between the moving grain and the packed grain bed.

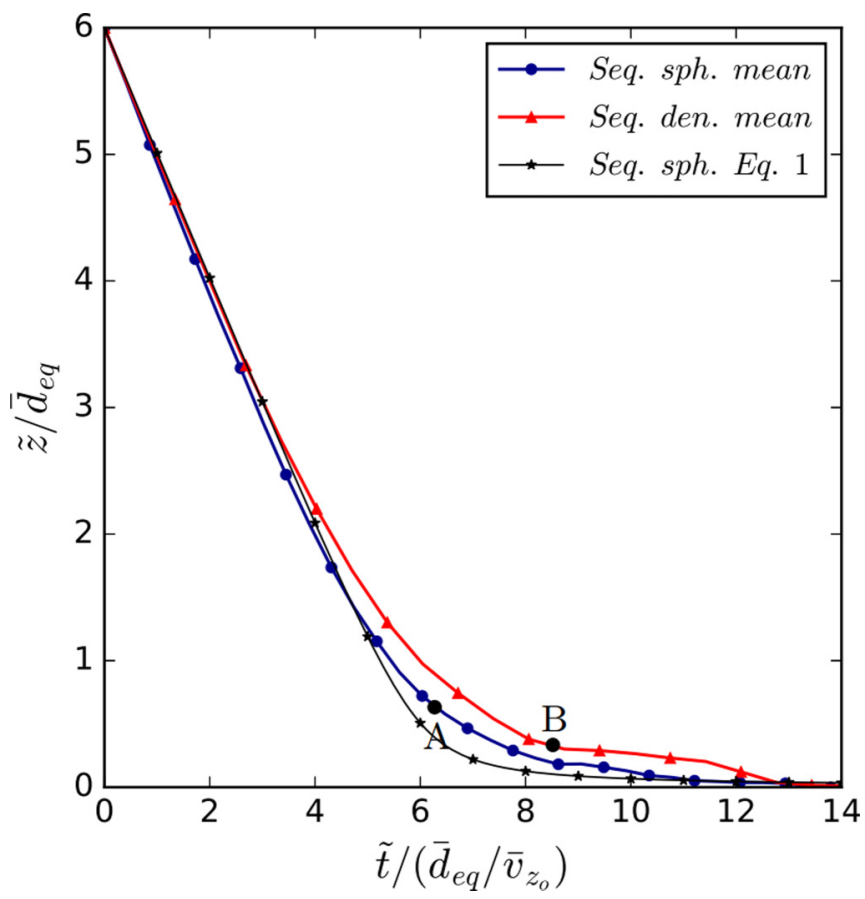

FIG. 5. Dimensionless vertical coordinate, $\tilde{z} / \bar{d}_{\mathrm{eq}}$, as a function of dimensionless time, $\tilde{t} /\left(\bar{d}_{\text {eq }} / \bar{v}_{z_{o}}\right)$ for spherical (o) and nonconvex dendritic grains $(\triangle)$ in sequential packing. The 10-particle mean trajectory is shown for each geometry. The theoretical solution of the trajectory of a smooth sphere approaching a smooth plane, given by Eq. (1), is added ( $\star$.

The solution of the trajectory of a sphere approaching a smooth plane, given by Eq. (1), is compared to the experimental trajectories. A good agreement is found between the trajectory of this simple model and that of the experiments for spherical grains, although the experimental trajectory is slightly smoother as a consequence of the irregular surface formed by the packed grain bed. The experimental trajectory of the nonconvex grains is smoother than that of the spherical grains which is a consequence of a larger dissipative lubrication force due to the grain geometry.

From now on, we focus on the collective case. The evolution of the vertical coordinate over time is shown in Fig. 6 for the spherical and the nonconvex dendritic grains. The mean dimensionless time to cross the final segment of the trajectory (corresponding to $0 \leqslant \tilde{z} \leqslant 6 \bar{d}_{\text {eq }}$ ) multiplies by a factor of around 2.3 for spherical grains and by a factor of around 3.5 for the nonconvex dendritic grains, when compared to their homologous cases in sequential packing.

The packing dynamics of the nonconvex dendritic grains is slower than that of the spherical grains. We observe that for the spherical grains it takes a mean dimensionless time of around $25\left(\bar{d}_{\mathrm{eq}} / \bar{v}_{z_{o}}\right)$ to cross the final segment of the trajectory (corresponding to $0 \leqslant \tilde{z} \leqslant 6 \bar{d}_{\mathrm{eq}}$ ), whereas for the nonconvex dendritic grains it takes $46\left(\bar{d}_{\mathrm{eq}} / \bar{v}_{z_{o}}\right)$.

Additionally, the instant of the first interparticle contact is also added for the spherical and nonconvex grains (see points $\mathrm{A}$ and $\mathrm{B}$ on the trajectories in Fig. 6). These 


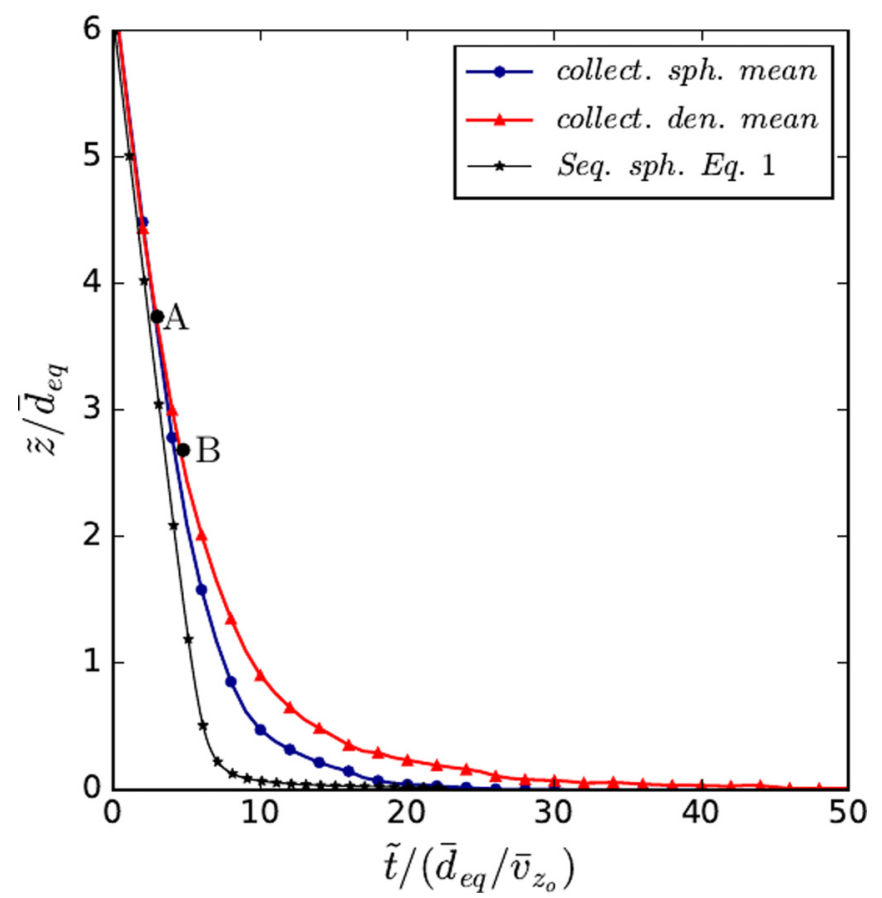

FIG. 6. Dimensionless vertical coordinate, $\tilde{z} / \bar{d}_{\mathrm{eq}}$, as a function of dimensionless time, $\tilde{t} /\left(\bar{d}_{\text {eq }} / \bar{v}_{z_{o}}\right)$ for spherical (o) and nonconvex dendritic grains $(\triangle)$ in collective packing. The 10-particle mean trajectory is shown for each geometry. The theoretical solution of the trajectory of a smooth sphere approaching a smooth plane, given by Eq. (1), is added ( $\star$ ).

instants are numerically obtained and widely discussed in Sec. IV C.

Figure 7 shows the dimensionless projected trajectory of the 10 tracked grains $\left\{\tilde{x} / \bar{d}_{\text {eq }}, \tilde{z} / \bar{d}_{\text {eq }}\right\}$ for the segment $0 \leqslant$ $\tilde{z} / \bar{d}_{\text {eq }} \leqslant 6$ for both grain collections in collective packing. We identify horizontal important displacements along the whole segment. When the grains are very close to their final packing positions, $0 \leqslant \tilde{z} / \bar{d}_{\text {eq }} \leqslant 1$, we identify zigzagging displacements. In the case of the nonconvex grain collection these horizontal displacements are more intense.

In Sec. III we showed the packing dynamics of sedimenting grains for a fixed hydrodynamic condition of low Stokes number, $\mathrm{St}=o\left(10^{-3}\right)$ for both sequential and collective packing protocols, obtained experimentally. We observed that the transition from the steady-state sedimentation until packing depends on the grain geometry. It takes considerably more time for the nonconvex dendritic grains with respect to the spherical grains for both packing protocols. The influence of the packing protocol (sequential or collective) is even more important than that of the grain geometry. For both spherical and nonconvex dendritic grains, the transition is much slower in case of collective packing compared to the sequential packing. This difference in the dynamics is attributed to collective interactions: interparticle collisions, lubrication grain-grain interactions and upward flow of ejected fluid. To complement the experimental work with information that we cannot access experimentally, the packing of sedimenting grains in case of collective packing protocol is numerically model in next section.
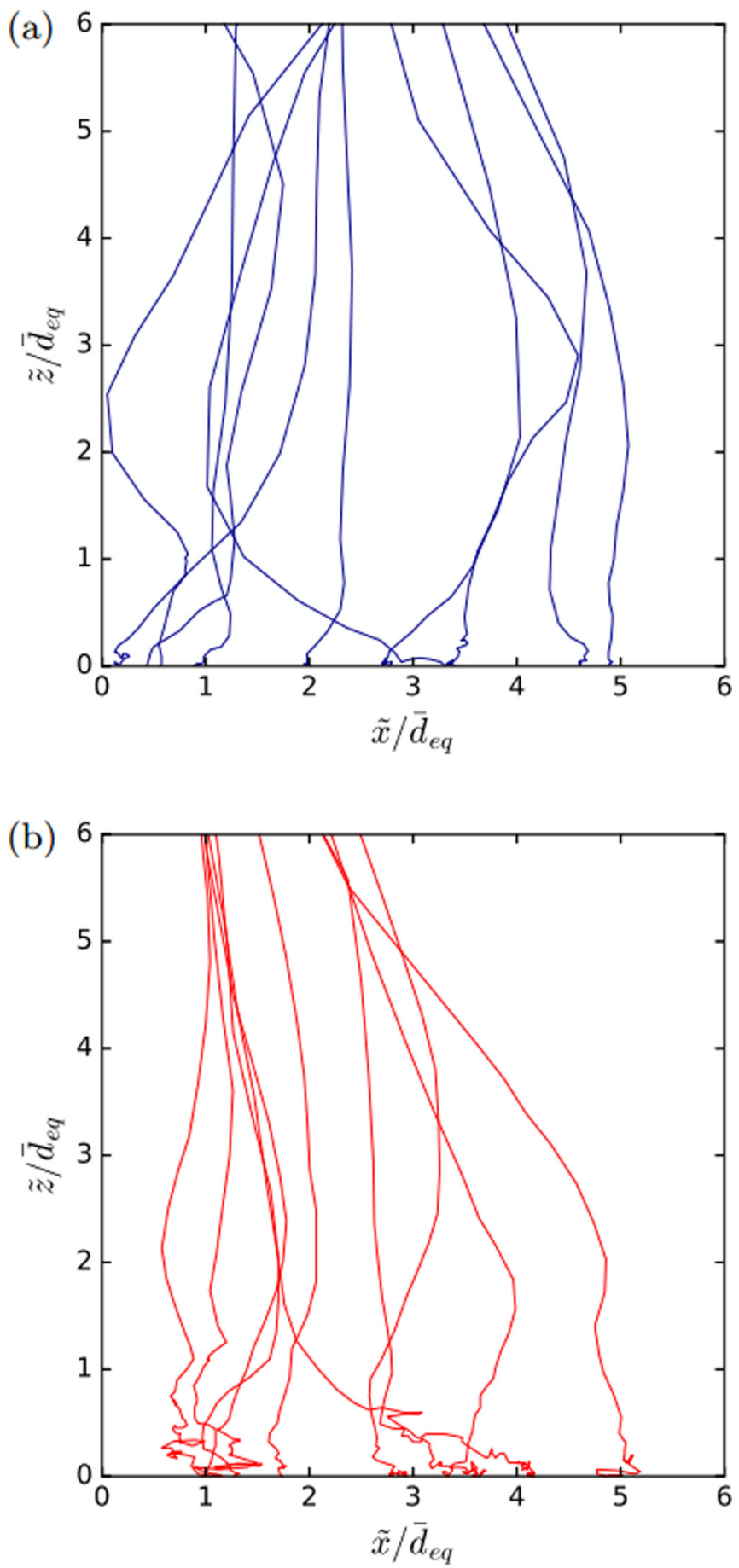

FIG. 7. Dimensionless projected trajectory $\left\{\tilde{x} / \bar{d}_{\mathrm{eq}}, \tilde{z} / \bar{d}_{\mathrm{eq}}\right\}$ for collective packing of spherical (a) and dendritic grains (b) obtained experimentally. Ten particle trajectories are shown for each geometry.

\section{DEM MODEL}

\section{A. Introduction}

The collective packing of sedimenting grains for both spherical and the nonconvex dendritic grains in glycerol is modeled by means of a DEM. By means of this numerical tool we can access to information of the collective grain dynamics that we cannot obtain experimentally, such as the time evolution of the local solid fraction, of the number of 
contacting neighbors, or of the grain rotation. In the model we account for both the translational and rotational degrees of freedom of the grains. The equations of the rigid body dynamics of each of the grains were already described in Ref. [24]. The interparticle collisions are accounted for by the linear spring-dashpot Kelvin-Voigt model, whereas the fluid dissipative effect over the grains is accounted for by the fluid buoyancy, drag force and moment, and lubrication forces between every couple of neighboring grains in the system.

In this model we do not solve the dynamics of the interstitial fluid between the grains which would require a fully coupled DEM-CFD model [25] with great limitations in the number of simulated grains and the domain size. Instead, the lubrication model is calibrated using the previous experimental work to account for the interstitial upflow dissipation on the grains. This calibration is explained along this section.

Herein we focus on the investigation of the time evolution of the local solid fraction, of the neighboring contacts and of the grain rotation during the transition in the Lagrangian specification for both the spherical and the dendritic grain collections at low Stokes hydrodynamic conditions of $\mathrm{St}=$ $o\left(10^{-3}\right)$.

\section{B. Model description for collective packing of sedimenting grains}

The simulation protocol of packing of sedimenting grains is designed to reproduce equivalent hydrodynamic conditions of St $=o\left(10^{-3}\right)$. We use a rectangular prism simulation domain of dimensions $12 d_{\mathrm{eq}} \times 12 d_{\mathrm{eq}} \times 140 d_{\mathrm{eq}}$ and $8 d_{\mathrm{eq}} \times$ $8 d_{\mathrm{eq}} \times 70 d_{\mathrm{eq}}$ for spherical and dendritic grains, respectively. Initially, the grain collections are uniformly distributed in their respective domains with $\phi_{\text {circ }}^{\text {sed }} \approx 0.20$ for both the sphere collection (composed by 2000 grains) and the nonconvex dendritic grain collection (composed by 400 grains). The nonconvex dendritic grains have the same geometry as in the experiments. The initial grain orientation is randomly computed [26]. The initial linear and angular grain velocities are null. We use periodic boundary conditions in the vertical domain walls, whereas we use a rigid wall at the bottom, $z=0$.

A density of $1400 \mathrm{~kg} / \mathrm{m}^{3}$ and an equivalent diameter of $7 \mathrm{~mm}$ were employed for both collections. The inertial tensor in body principal axes is computed as $\mathbf{I}_{o, b}=\frac{\pi}{60} \rho_{\mathrm{s}} d_{\mathrm{eq}}^{5} \mathbf{1}$ where $\mathbf{1}$ is the unit tensor of rank 2, for both spherical and dendritic grains. We apply the apparent weight (difference between the grain weight and the fluid buoyancy) to each grain, $\mathbf{F}_{\mathrm{w}-\mathrm{b}}=\left(\rho_{s}-\rho_{l}\right) \frac{\pi}{6} d_{\mathrm{eq}}^{3} \mathbf{g}_{o}$, with the gravity, $\mathbf{g}_{o}$, antiparallel to the direction $z$. Assuming the Stokes regime and neglecting the multi-grain effect, the drag force is given by $\mathbf{F}_{d}=-A_{d} \rho_{l} v_{l}\left(d_{\mathrm{eq}} / 2\right) \mathbf{v}_{o}$ and the drag moment is given by $\mathbf{M}_{o, d}=-D_{d} \rho_{l} v_{l}\left(d_{\mathrm{eq}} / 2\right)^{3} \omega_{o}$ with $\mathbf{v}_{o}$ and $\omega_{o}$ the linear and angular grain velocities. The $A_{d}$ and $D_{d}$ drag coefficients are $6 \pi$ and $8 \pi$, respectively, for both spherical and nonconvex dendritic geometries. The fluid density and dynamic viscosity are $1260 \mathrm{~kg} / \mathrm{m}^{3}$ and $1.2 \mathrm{~Pa} \mathrm{~s}$, respectively. Under these conditions, the grains quickly reach a steady-state sedimentation velocity value.
Additionally, a model of lubrication forces between every couple of neighboring grains is included based on the model presented in Ref. [27]. Originally this model was developed for monodisperse sphere collections in quiescent fluid. The lubrication forces between two neighboring grains $i$ and $j$ are implemented as distance forces of normal, $\mathbf{F}_{\text {lub }, i j, n}$, and tangential components, $\mathbf{F}_{\text {lub }, i j, t}$ :

$$
\begin{gathered}
\mathbf{F}_{\text {lub }, i j, n}=\lambda_{\text {lub }} \frac{3}{8} \pi \rho_{l} v_{l} \frac{d_{\mathrm{eq}}^{2}}{h} \Delta \mathbf{v}_{i j} \mathbf{e}_{n_{i j}}, \\
\mathbf{F}_{\text {lub }, i j, t}=\lambda_{\text {lub }} \frac{\pi}{2} \rho_{l} v_{l} d_{\text {eq }}\left\{\left(1+\frac{h}{d_{\mathrm{eq}}}\right)\right. \\
\left.\times \ln \left(1+\frac{d_{\mathrm{eq}}}{h}\right)-1\right\} \Delta \mathbf{v}_{i j} \mathbf{e}_{t_{i j}},
\end{gathered}
$$

where $h, \Delta \mathbf{v}_{i j}, \mathbf{e}_{n_{i j}}$ and $\mathbf{e}_{t_{i j}}$ read

$$
\begin{gathered}
h=\left|\mathbf{r}_{j}-\mathbf{r}_{i}\right|-2 d_{\mathrm{lub}}^{*} d_{\mathrm{eq}}, \\
\Delta \mathbf{v}_{i j}=\mathbf{v}_{j}-\mathbf{v}_{i}, \\
\mathbf{e}_{n_{i j}}=\frac{\mathbf{r}_{j}-\mathbf{r}_{i}}{\left|\mathbf{r}_{j}-\mathbf{r}_{i}\right|}, \\
\mathbf{e}_{t_{i j}}=\frac{\Delta \mathbf{v}_{i j}-\left(\Delta \mathbf{v}_{i j} \mathbf{e}_{n_{i j}}\right) \mathbf{e}_{n_{i j}}}{\left|\Delta \mathbf{v}_{i j}-\left(\Delta \mathbf{v}_{i j} \mathbf{e}_{n_{i j}}\right) \mathbf{e}_{n_{i j}}\right|} .
\end{gathered}
$$

We modified the original lubrication model from Ref. [27] by the addition of two new dimensionless parameters: $\lambda_{\text {lub }}$ and $d_{\text {lub }}^{*}$. In the original lubrication model they are $\lambda_{\text {lub }}=1$ and $d_{\text {lub }}^{*}=1$. The values of these two parameters are calibrated by means of the experimental mean trajectory (Fig. 6) to account for the dissipative effect of the fluid upflow on the grains (by means of the parameter $\lambda_{\text {lub }}$ ) and the importance of the lubrication force for the nonspherical nonconvex grains (by means of the parameter $\left.d_{\text {lub }}^{*}\right)$.

We use the classical Kelvin-Voigt (spring-dashpot) contact model for the normal component of the contact with a clumped-logic discretization of the nonspherical dendritic grains as described in Ref. [24]. The frictional contact is not considered in this paper. The values of the spring stiffness are chosen to avoid grain overlaps larger than $0.01 d_{\mathrm{eq}}$. The critical damping is chosen for the linear dashpot.

\section{Simulation results and discussion}

In Figs. 8(a) and 8(b), a 3D view of the sedimentation and packing simulations of spherical and nonconvex dendritic grain collections is shown, respectively.

Only the parameter $\lambda_{\text {lub }}$ is calibrated for the spherical geometry whereas both $\lambda_{\text {lub }}$ and $d_{\text {lub }}^{*}$ are calibrated for the nonconvex dendritic geometry by means of the experimental mean trajectory of 10 grains (see Fig. 6). In Figs. 9(a) and 9(b) we show the 10-grain mean experimental trajectory compared to the 10-grain mean numerical trajectory for several values of the parameters $\lambda_{\text {lub }}$ and $d_{\text {lub }}^{*}$. The best calibration with our model is achieved with $\lambda_{\text {lub }}$ of approximately 15 for spheres, whereas $\lambda_{\text {lub }}$ of approximately 15 and $d_{\text {lub }}^{*}=1.4$ for nonconvex dendritic grains. 
(a)

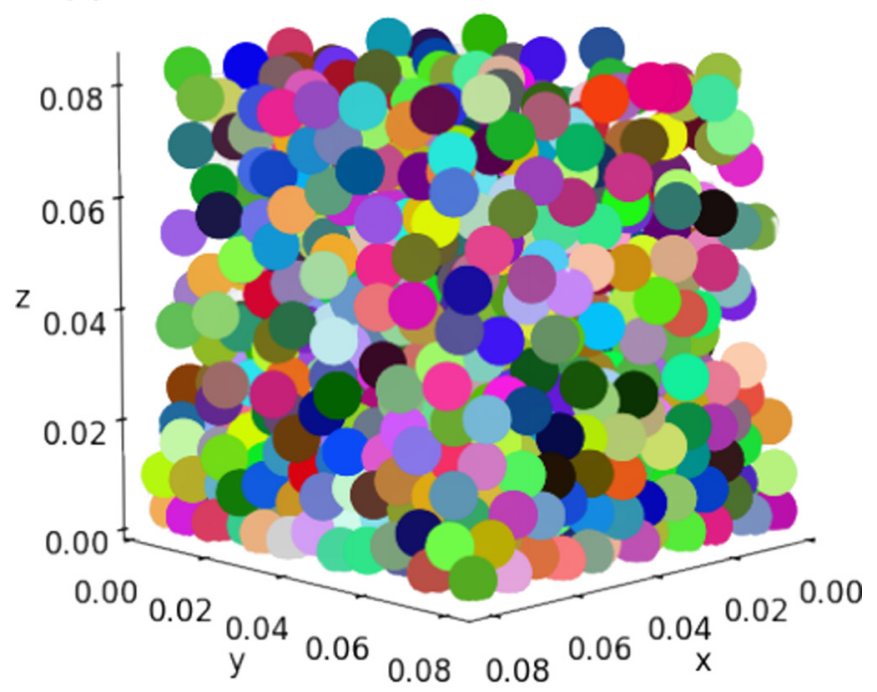

(b)

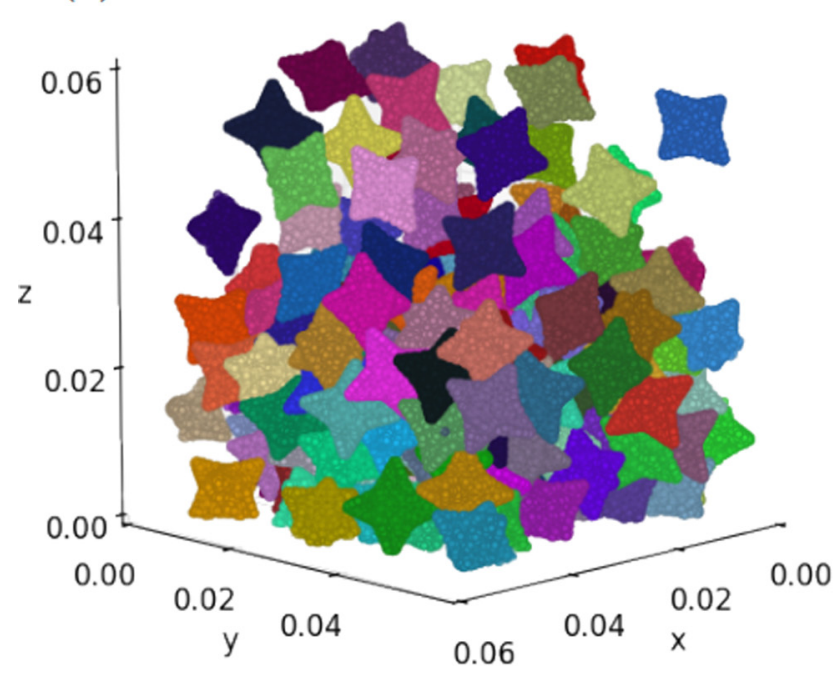

FIG. 8. Three-dimensional snapshot of the DEM calibrated simulations of the collective sedimentation and packing in glycerol for (a) spherical grains and (b) nonconvex dendritic grains.

After calibrating the model to obtain a similar packing dynamics as that of the experimental collective cases, a comparison of the average packing fraction from the calibrated numerical simulations, $\left\langle\phi_{s}\right\rangle_{\Omega_{\text {pack }}}^{\text {dem }}$, and the average packing fraction from the packing experiments in glycerol [14], $\left\langle\phi_{s}\right\rangle_{\Omega_{\mathrm{pack}}}^{\exp }$, is carried out for both spherical and dendritic grains in Table I to ensure that this calibration does not have an effect on the

TABLE I. Numerical-experimental comparison: Packing fraction in glycerol.

\begin{tabular}{lccr}
\hline \hline Grain collection & $\left\langle\phi_{s}\right\rangle_{\Omega_{\mathrm{pack}}}^{\mathrm{dem}}[\%]$ & $\left\langle\bar{\phi}_{s}\right\rangle_{\Omega_{\mathrm{pack}}}^{\exp }[\%]$ & $\epsilon_{\phi_{s}}[\%]$ \\
\hline Spherical & 60.3 & $57.5 \pm 0.8$ & 4.9 \\
Nonconvex dendritic & 37.5 & $39.7 \pm 1.3$ & -5.5 \\
\hline \hline
\end{tabular}
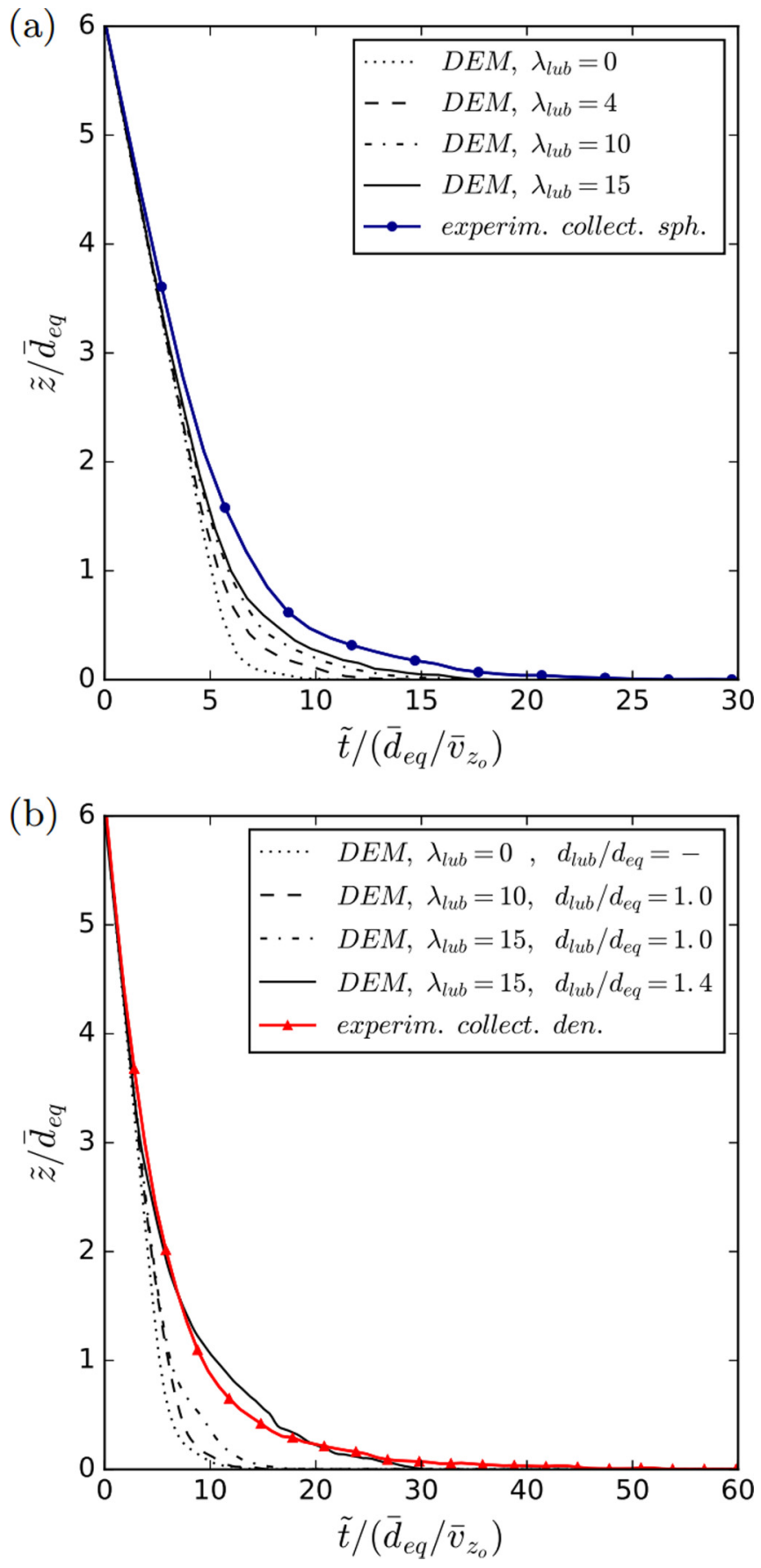

FIG. 9. Numerical and experimental comparison of a 10-particle mean vertical trajectory. A parametrical study of the numerical trajectory is presented. The spherical and dendritic geometry results are presented in (a) and (b), respectively.

final packing. A relative error of $4.9 \%$ and $-5.5 \%$ is found for spherical and nonconvex dendritic grains, respectively. In this way, we determine that the lubrication model employed to mimic the packing dynamics found in the experimental work also leads to an accurate prediction of the packing fraction of the system.

In Fig. 10 we show the dimensionless projected trajectory of 10 tracked grains $\left\{\tilde{x} / \bar{d}_{\mathrm{eq}}, \tilde{z} / \bar{d}_{\mathrm{eq}}\right\}$ for both grain collections that results from our DEM simulations. Equivalently to the 

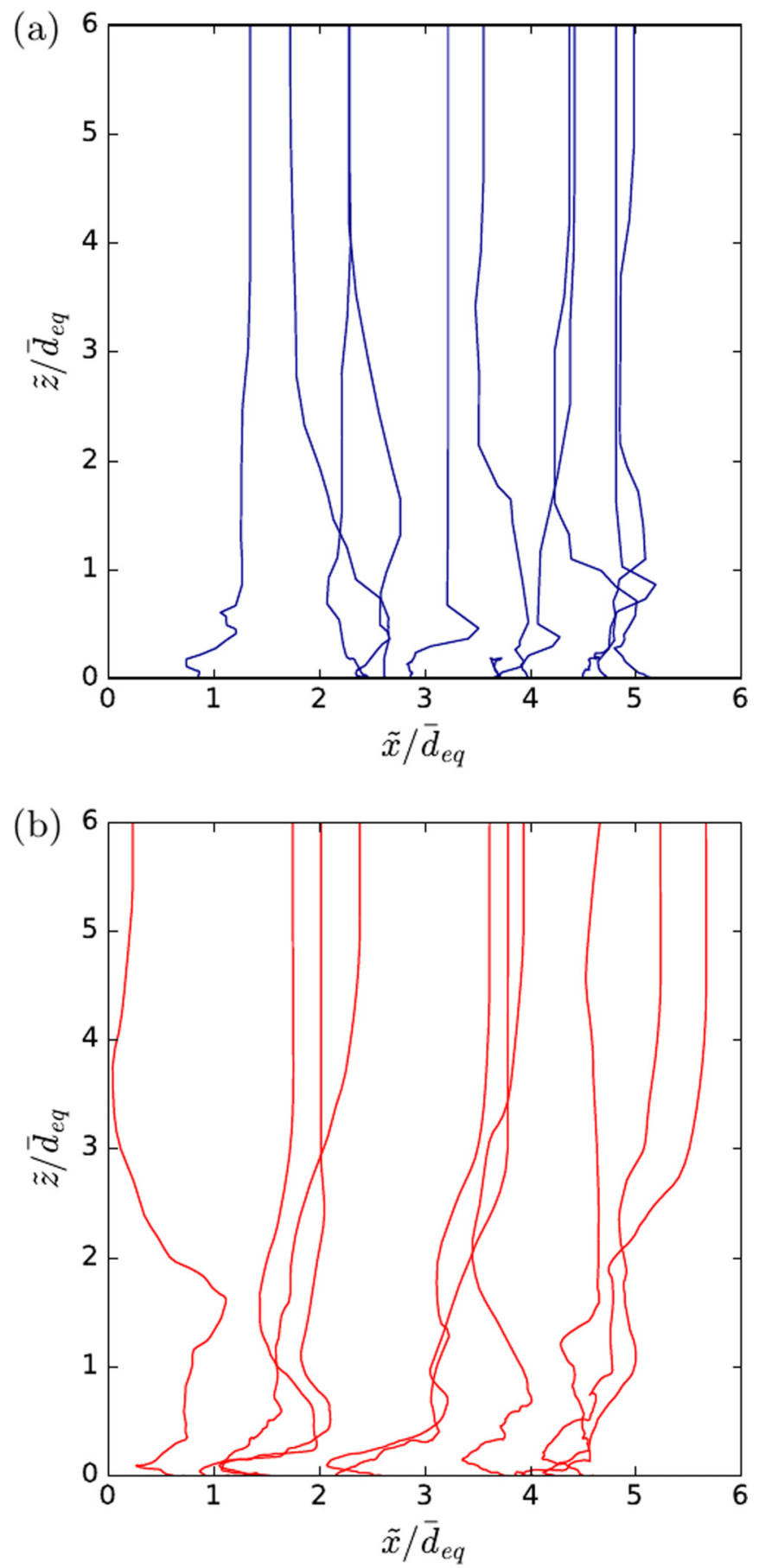

FIG. 10. Simulated projected trajectory $\left\{\tilde{x} / \bar{d}_{\text {eq }}, \tilde{z} / \bar{d}_{\text {eq }}\right\}$ for spherical (a) and dendritic grains (b) collective packing. Ten particle trajectories are shown for each geometry.

experimental homologous results in Fig. 7, with the DEM model we obtain that the horizontal displacements of the grains becomes important when they are close to pack. For the nonconvex grains this effect is more intense. According to the interparticle collision results that we later show in Fig. 14, below a mean vertical coordinate of $\tilde{z} / \bar{d}_{\text {eq }} \approx 3.8$ for spheres and 2.7 for nonconvex dendritic grains, both interparticle collisions and lubrication interactions between neighboring

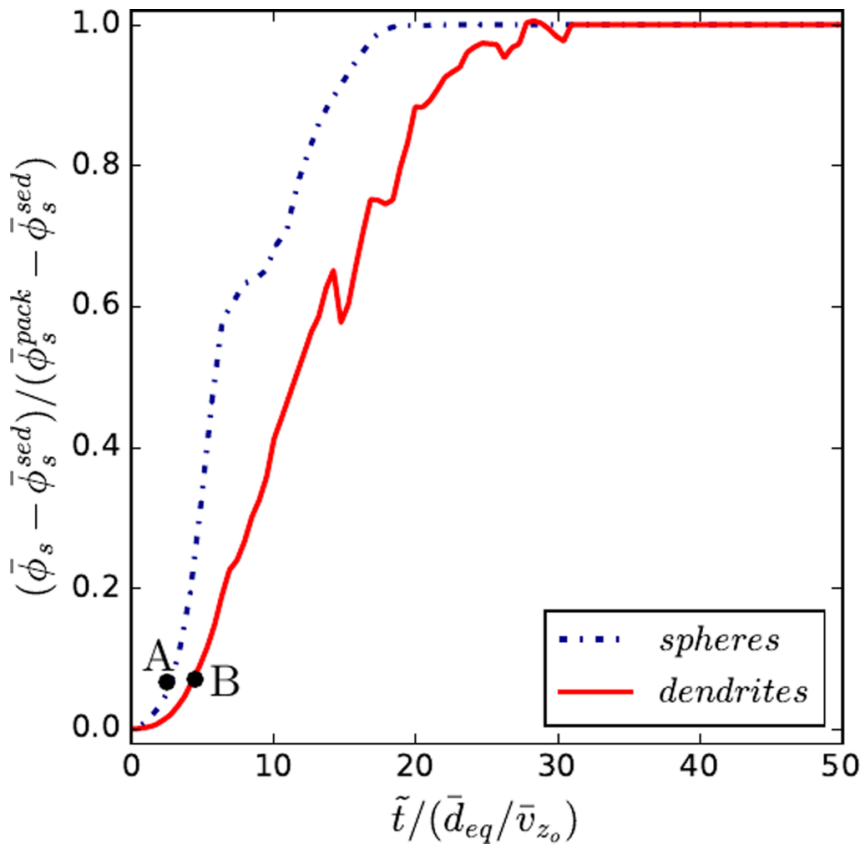

FIG. 11. Evolution of the 10-grain mean normalized local solid fraction over the dimensionless time for the spherical grain collection (dashed line) and the dendritic grain collection (solid line) at low Stokes conditions, $\mathrm{St}=o\left(10^{-3}\right)$. The time origin is that of the Figs. 6 and 9 .

grains occur. Above it, only lubrication interactions between neighboring grains are expected.

Subsequently, we present the time evolution and $z$ evolution of the local solid fraction, of the number of contacting neighbors, and of the grain rotation in Lagrangian specification in Figs. 11-16 for both the spherical and the dendritic grain collections, where the time origin in these time evolutions is the same than that in Figs. 6 and 9.

By means of these three variables we are able to better understand how the sedimenting grains decelerate and rearrange until the packing is reached, identifying important parameters of the packing dynamics such as the characteristic size of the transition region, the characteristic time of the grains in the transition region, i.e., the time the grains spend between the steady-state regime and the final mechanical equilibrium, and the instant when the interparticle collision begin important, among others.

The local solid fraction is computed every $2 \mathrm{~s}$ with the Voronoi tessellation for all the grains of the system using the VORO++ open library [28]. The mean solid local fraction, $\bar{\phi}_{s}$, over $n_{\text {track }}$ grains for an instant $t$ is given by

$$
\bar{\phi}_{s}(t)=\frac{1}{n_{\text {track }}} \sum_{i=1}^{n_{\text {track }}} \frac{\frac{\pi}{6} d_{\mathrm{eq}}^{3}}{V_{\text {celli }}(t)},
$$

where the number of tracked grains, $n_{\text {track }}$, is 10 (equal to experiments), and $V_{\text {cell }}(t)$ is the Voronoi cell of the grain $i$ at the instant $t$. For a better comparison of the local solid fraction during the packing dynamics between the spherical and the nonconvex dendritic grains, we show the normalized value of the mean local solid fraction. The mean local solid fraction during the steady-state sedimentation, $\bar{\phi}_{s}^{\text {sed }}$, is 0.20 and 0.05 , 


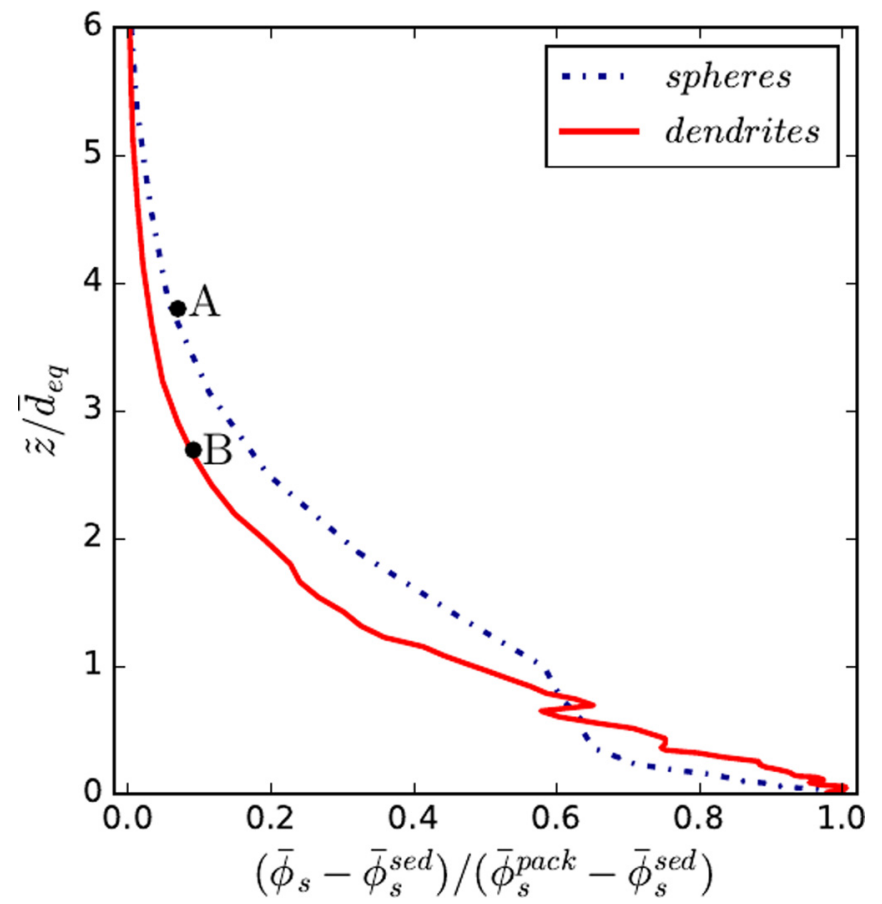

FIG. 12. Lagrangian $z$ evolution of the 10-grain mean normalized local solid fraction for the spherical grain collection (dashed line) and the dendritic grain collection (solid line) at low Stokes conditions, $\mathrm{St}=o\left(10^{-3}\right)$. The time origin is that of the Figs. 6 and 9 .

for spherical and dendritic grains, respectively, whereas the mean local solid fraction at packing for the 10 tracked grains, $\bar{\phi}_{s}^{\text {pack }}$, is 0.594 and 0.362 , for spherical and dendritic grains, respectively.

In Figs. 11 and 12 we show the time evolution and $z$ evolution of the local solid fraction, respectively. In Fig. 11 the local solid fraction initiates to increment from its initial value of steady-state sedimentation for a dimensionless time that corresponds approximately to zero, i.e., $\tilde{z} / \bar{d}_{\mathrm{eq}} \approx 6$. We establish the onset of the transition from sedimentation to packing at this point for both spherical and nonconvex dendritic grains for the collective packing protocol. The packing value of the solid fraction is reached for a dimensionless time of around 20 and 30 for spherical and nonconvex dendritic grains, respectively.

For the collective packing protocol, we also subdivided the transition from sedimentation to packing into stages I and II. The grains decelerate without interparticle collisions during stage I. The onset of this phase is the onset of the transition. Stage II is characterized by the interparticle collisions. The instant of switch between stages I and II is determined by the first contact among the grains (which is shown later in Figs. 13 and 14). The end of stage II is the end of the transition, i.e., when the mechanical equilibrium (packing) is achieved. The instant of switch between the phases I and II is indicated by the points A and B for the spherical and nonconvex dendritic grains, respectively, in Figs. 11 and 12.

In Figs. 13 and 14 we show the time evolution and $z$ evolution of the mean number of contacting neighbors, respectively. The contacting neighbors of a certain grain i, $N_{c_{i}}$, are those grains around $i$ that have a nonnull contact force with

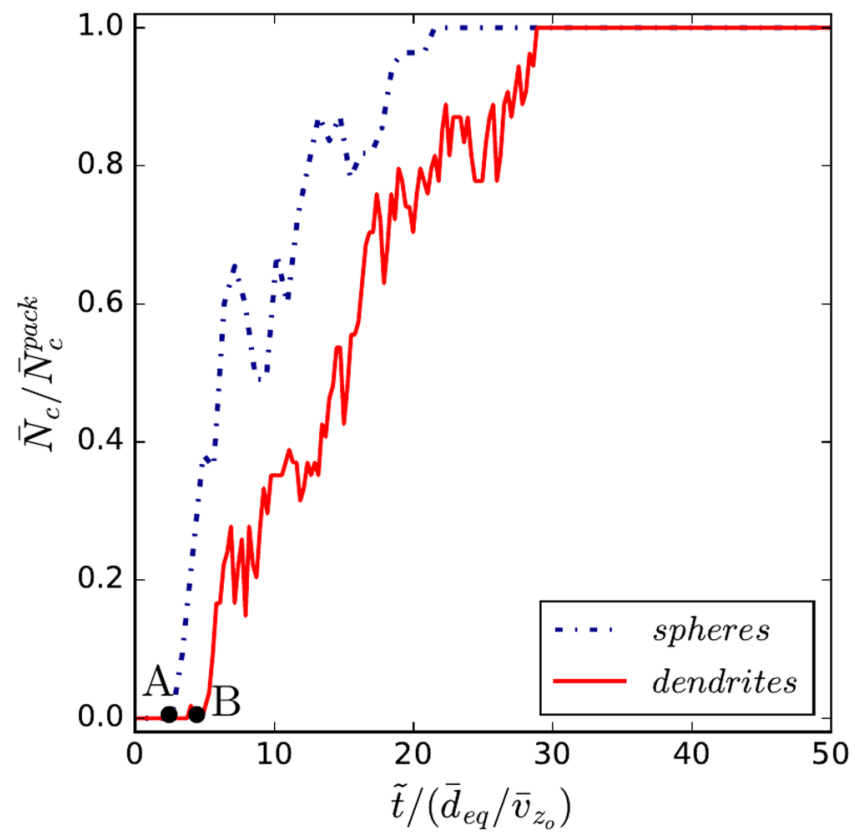

FIG. 13. Evolution of the 10-grain mean normalized number of contacting neighbors over the dimensionless time for the spherical grain collection (dashed line) and the dendritic grain collection (solid line) at low Stokes conditions, $\mathrm{St}=o\left(10^{-3}\right)$. The time origin is that of the Figs. 6 and 9.

$i$. For spherical grains the number of contacting neighbors is equivalent to the number of contacts; however, for nonconvex grains a pair of neighbors can share multiple contacts (up to three in case of our nonconvex grains). The mean number of

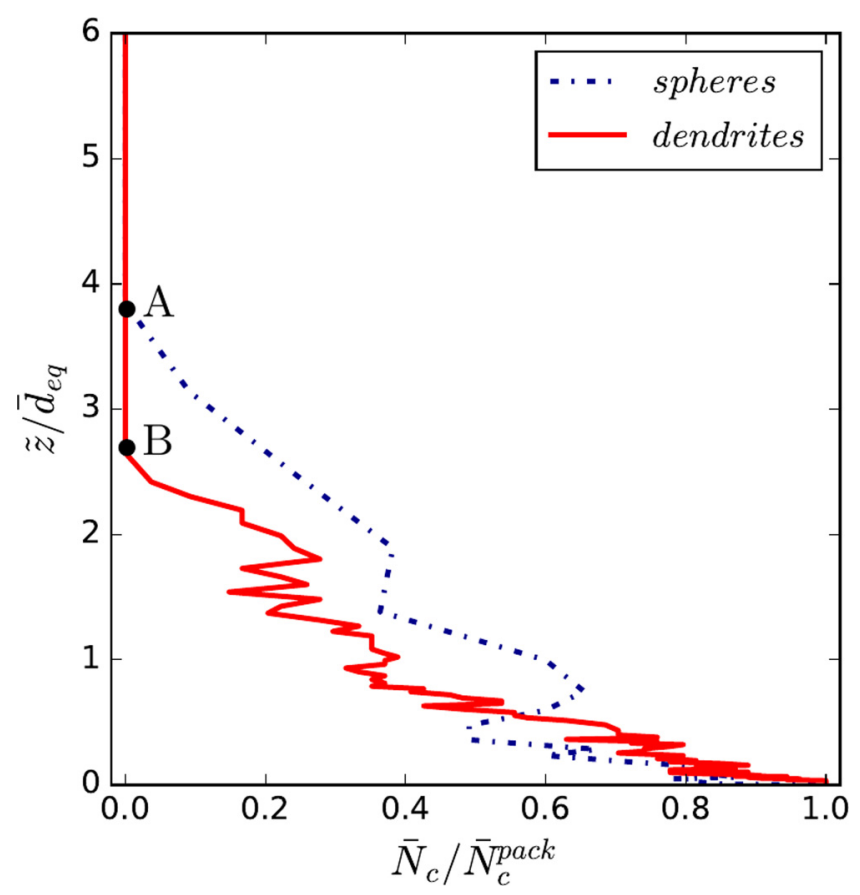

FIG. 14. Lagrangian $z$ evolution of the 10-grain mean normalized number of contacting neighbors for the spherical grain collection (dashed line) and the dendritic grain collection (solid line) at low Stokes conditions, $\mathrm{St}=o\left(10^{-3}\right)$. 


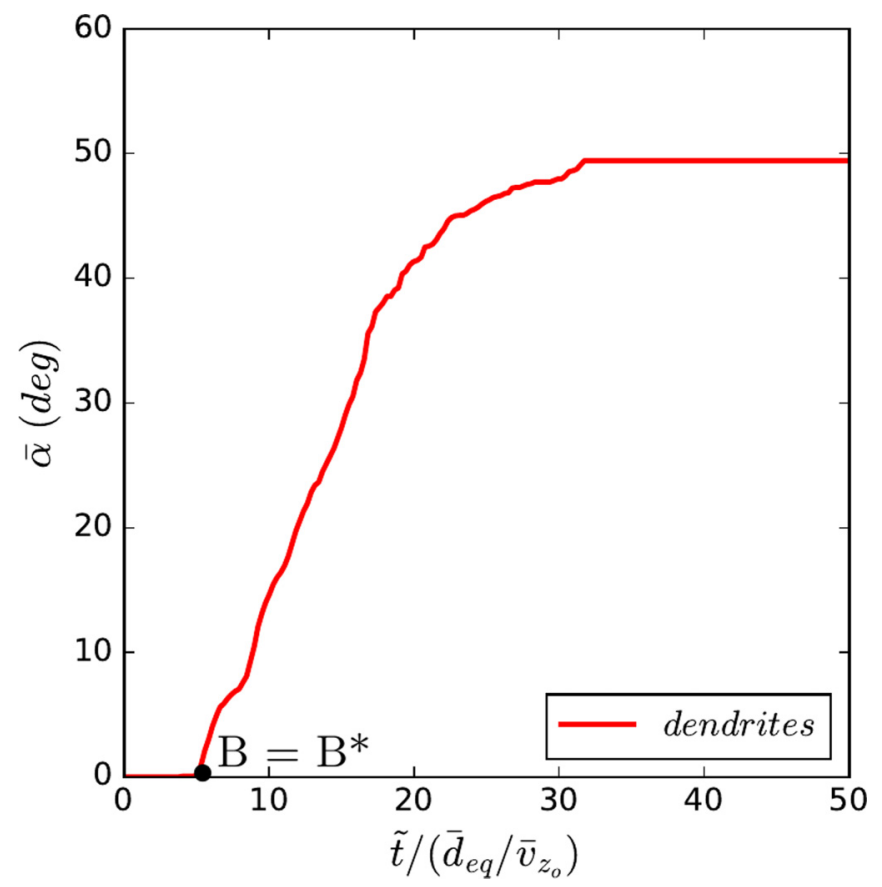

FIG. 15. Evolution of the 10-particle mean grain rotation over the dimensionless time for the dendritic grain collection (solid line) at low Stokes conditions, $\mathrm{St}=o\left(10^{-3}\right)$. The time origin is that of the Figs. 6 and 9. The spherical grain rotation is identically null.

contacting neighbors, $\bar{N}_{c}$, over $n_{\text {track }}$ grains for an instant $t$ is given by

$$
\bar{N}_{c}(t)=\frac{1}{n_{\text {track }}} \sum_{i=1}^{n_{\text {track }}} N_{c_{i}}(t),
$$

We identify the instant when the interparticle collisions begin at the points $\mathrm{A}$ and $\mathrm{B}$ for the spherical and nonconvex grains, respectively. Before this instant, the grains move without collisions. The point B appears with a delay with respect to A since tip-to-tip contact between nonconvex dendritic grains is not very common, i.e., they generally enter in contact while their circumscribing spheres overlap.

The mean number of contacting neighbors increases from 0 up to its value at packing, $\bar{N}_{c}^{\text {pack }}$. For the 10 tracked grains we obtained a value of $\bar{N}_{c}^{\text {pack }}=5.5$ for the spheres, which is slightly lower than the value of approximately six neighboring contacts found for sedimentation and packing of frictionless spheres in Ref. [29], whereas, we obtained $\bar{N}_{c}^{\text {pack }}=5.4$ for the dendritic grains, which is sligher lower than the 6.3 contacting neighbors found by Ref. [30] for dry packing of tetrahedrons (nonspherical convex grains).

To understand the role of the grain rotation during the packing dynamics, the mean grain rotation, $\bar{\alpha}$, is investigated and presented in Figs. 15 and 16. It is given, for each of the tracked grains, by

$$
\alpha(\tilde{t})=\int_{\tau=0}^{\tau=\tilde{t}}|\omega(\tau)| d \tau .
$$

In our simulations, the grains are frictionless, which is reasonable under wet contact conditions. With this hypothesis,

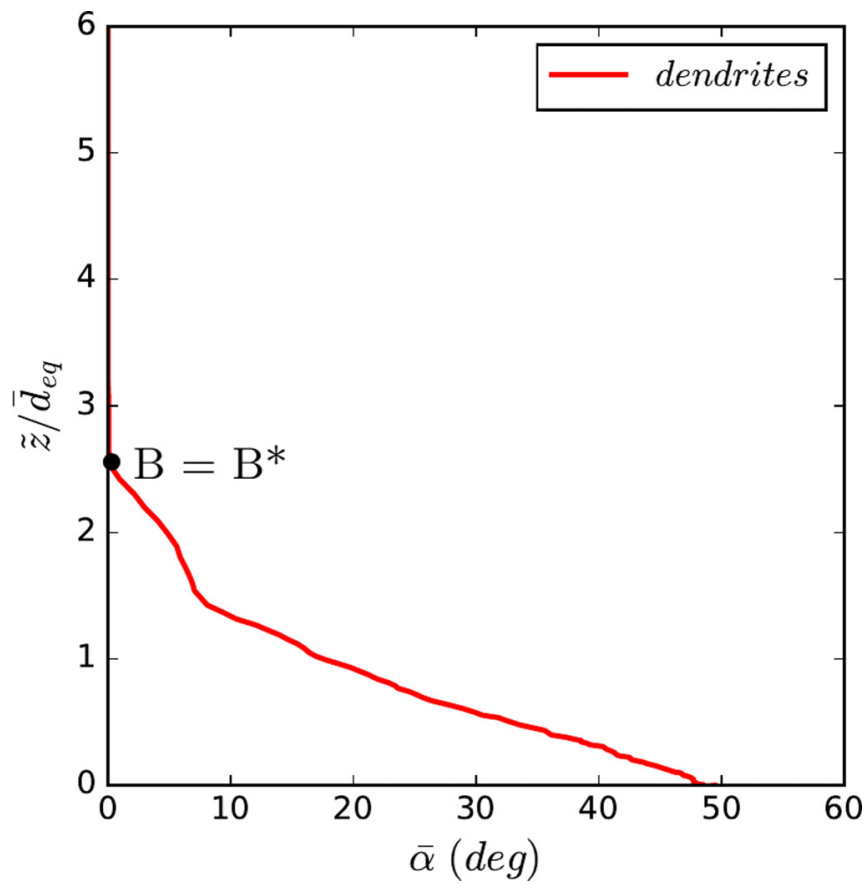

FIG. 16. Lagrangian $z$ evolution of the 10-particle mean grain rotation for the dendritic grain collection (solid line) at low Stokes conditions, $\mathrm{St}=o\left(10^{-3}\right)$. The spherical grain rotation is identically null.

we find that the spherical grain rotation is identically null whereas the nonconvex dendritic grains have an important mean rotation during the packing dynamics (up to 50 degrees). This result is exclusively a consequence of the nonspherical geometry. In the collision between two frictionless spheres, the point of contact is aligned with their centers, so the moment due to contact is null. In the case of a collision between a couple of nonspherical grains, the point of contact is not generally aligned with their centers, so the moment due to contact is nonnull with the particle rotation as consequence of it.

In Fig. 15 we show the time evolution of the 10-mean grain rotation for the nonconvex dendritic grain collection. The point $\mathrm{B}^{*}$ is the instant when the rotation begins. After it the mean rotation increases up to its final packing value. The point $\mathrm{B}^{*}$ is coincident with the point $\mathrm{B}$ since the rotation is a consequence of the interparticle collisions. In Fig. 16 we show the $z$ evolution of the 10-mean grain rotation for the nonconvex grain collection. We observe that the translational degrees of freedom are enough to form the packing network in case of spherical grains, whereas, the nonconvex dendritic grains interlace forming the packing structure with both translational and rotational degrees of freedom strongly coupled once the interparticle collision begins.

\section{CONCLUSIONS}

The phenomenon of packing formation by sedimenting grains is investigated in the context of solidification of metal alloys. We focused on the investigation of the packing dynamics, i.e., the transition between the grain steady-state 
sedimentation regime and the final mechanical equilibrium -packing- where the grains decelerate and rearrange. Low Stokes hydrodynamic conditions of $\mathrm{St}=o\left(10^{-3}\right)$ are considered which are typical in solidification. The sedimenting grains are considered growthless assuming that grain growth velocity is negligible with respect to the grain sedimentation velocity. Two grain geometries are investigated: spheres and six-arm nonconvex grain collections which represent the globular and dendritic characteristic grain geometries in solidification, respectively.

By means of the combination of an experimental and a numerical approaches, the packing dynamics is investigated for both the spherical and nonconvex collections under two different protocols (sequential and collective sedimentation), providing a better understanding of how the sedimenting grains decelerate and rearrange to form the packing network.

The vertical segment length traveled by the grains during the transition from sedimentation to packing is identified to be lower than $6 d_{\text {deq }}$ for both protocols and geometries, which gives an approximation of the characteristic size of the transition in the solidification context.

We show that the grain collectivity plays a much important role than the grain geometry on the packing dynamics. The collective effects such as the grain-grain interactions via lubrication effects, collisions and upward oriented liquid flow considerably increase the time that grains spend between the steady-state sedimentation and the final mechanical equilibrium.

Two stages for both protocols are identified: a first stage of deceleration of the grains only governed by fluid dissipative effects, and a late stage where the grain collisions are also fundamental governing the grain deceleration and rearrangement until the packing is achieved. In the collective case, the second stage becomes predominant.

We show that both translational and rotational degrees of freedom are fundamental for the formation of the packing network in case of the nonconvex dendritic grains whereas in case of the spherical collection the translational displacements are enough.

Further work corresponds to investigate the packing dynamics for more collective cases and Stokes conditions as well as including the effect of the grain growth during the packing dynamics. Additionally, the results provided in this work can be applied to improve the current modeling tools of industrial casting processes.

\section{ACKNOWLEDGMENTS}

This work was supported by the French State through the program "Investment in the future" operated by the National Research Agency (ANR) and referenced by ANR-11 LABX0008-01 (LabEx DAMAS).
[1] M. Založnik, A. Kumar, H. Combeau, M. Bedel, P. Jarry, and E. Waz, Adv. Eng. Mater. 13, 570 (2011).

[2] H. Combeau, M. Založnik, S. Hans, and P. E. Richy, Metall. Mater. Trans. B 40, 289 (2009).

[3] J. A. Dantzig and M. Rappaz, Solidification, 1st ed. (EPFL Press, Lausanne, Switzerland, 2009).

[4] G. Y. Onoda and E. G. Liniger, Phys. Rev. Lett. 64, 2727 (1990).

[5] M. Jerkins, M. Schröter, H. L. Swinney, T. J. Senden, M. Saadatfar, and T. Aste, Phys. Rev. Lett. 101, 018301 (2008).

[6] M. Schröter, D. I. Goldman, and H. L. Swinney, Phys. Rev. E 71, 030301 (2005).

[7] T. Aste, T. Di Matteo, M. Saadatfar, T. J. Senden, M. Schröter, and H. L. Swinney, Europhys. Lett. 79, 24003 (2007).

[8] G. W. Delaney, T. Di Matteo, and T. Aste, Soft Matter 6, 2992 (2010).

[9] G. R. Farrell, K. M. Martini, and N. Menon, Soft Matter 6, 2925 (2010).

[10] J. Baker and A. Kudrolli, Phys. Rev. E 82, 061304 (2010).

[11] A. D. Rakotonirina, J.-Y. Delenne, and A. Wachs, in Proceedings of the International Conference on Micromechanics of Granular Media (Powders \& Grains), EPJ Web of Conferences Vol. 140 (EDP Sciences, Montpellier, France, 2017).

[12] J. Barés, Y. Zhao, M. Renouf, K. Dierichs, and R. Behringer, EPJ Web Conf. 140, 06021 (2017).

[13] I. Malinouskaya, V. V. Mourzenko, J.-F. Thovert, and P. M. Adler, Phys. Rev. E 80, 011304 (2009).
[14] A. Olmedilla, M. Založnik, B. Rouat, and H. Combeau, Phys. Rev. E 97, 012910 (2018).

[15] A. Mongruel, C. Lamriben, S. Yahiaoui, and F. Feuillebois, J. Fluid Mech. 661, 229 (2010).

[16] F.-L. Yang and M. L. Hunt, Phys. Fluids 18, 121506 (2006).

[17] E. Izard, T. Bonometti, and L. Lacaze, J. Fluid Mech. 747, 422 (2014).

[18] H. Brenner, Chem. Eng. Sci. 16, 242 (1961).

[19] R. G. Cox and H. Brenner, Chem. Eng. Sci. 22, 1753 (1967).

[20] N. Lecoq, R. Anthore, B. Cichocki, P. Szymczak, and F. Feuillebois, J. Fluid Mech. 513, 247 (2004).

[21] K. Dong, R. Yang, R. Zou, and A. Yu, AIChE J. 58, 1409 (2012).

[22] S.-1. Xu, R. Sun, Y.-q. Cai, and H.-1. Sun, Granular Matter 20, 4 (2017).

[23] N.-S. Cheng, Ind. Eng. Chem. Res. 47, 3285 (2008).

[24] A. Olmedilla, M. Založnik, and H. Combeau, EPJ Web Conf. 140, 06002 (2017).

[25] E. Izard, T. Bonometti, and L. Lacaze, J. Comput. Multiphase Flows 6, 391 (2014).

[26] K. Shoemake, Comput. Graphics 19, 245 (1985).

[27] N. Frankel and A. Acrivos, Chem. Eng. Sci. 22, 847 (1967).

[28] C. H. Rycroft, Chaos 19, 041111 (2009).

[29] G. W. Delaney, J. E. Hilton, and P. W. Cleary, Phys. Rev. E 83, 051305 (2011).

[30] A. Jaoshvili, A. Esakia, M. Porrati, and P. M. Chaikin, Phys. Rev. Lett. 104, 185501 (2010). 